\title{
CHARACTERIZATION OF SUPER-RADIAL GRAPHS
}

\author{
KM. KATHIRESAN \\ Center for Research and Post Graduate Studies in Mathematics \\ Ayya Nadar Janaki Ammal College \\ Sivakasi-626 124, Tamil Nadu, India \\ e-mail: kathir2esan@yahoo.com \\ G. Marimuthu \\ Department of Mathematics \\ The Madura College \\ Madurai-625 011, Tamil Nadu, India \\ e-mail: yellowmuthu@yahoo.com \\ AND \\ C. Parameswaran \\ Center for Research and Post Graduate Studies in Mathematics \\ Ayya Nadar Janaki Ammal College \\ Sivakasi-626 124, Tamil Nadu, India \\ e-mail: parames65_c@yahoo.com
}

\begin{abstract}
In a graph $G$, the distance $d(u, v)$ between a pair of vertices $u$ and $v$ is the length of a shortest path joining them. The eccentricity $e(u)$ of a vertex $u$ is the distance to a vertex farthest from $u$. The minimum eccentricity is called the radius, $r(G)$, of the graph and the maximum eccentricity is called the diameter, $d(G)$, of the graph. The super-radial graph $R^{*}(G)$ based on $G$ has the vertex set as in $G$ and two vertices $u$ and $v$ are adjacent in $R^{*}(G)$ if the distance between them in $G$ is greater than or equal to $d(G)-r(G)+1$ in $G$. If $G$ is disconnected, then two vertices are adjacent in $R^{*}(G)$ if they belong to different components. A graph $G$ is said to be a super-radial graph if it is a super-radial graph $R^{*}(H)$ of some graph $H$. The main objective of this paper is to solve the graph equation $R^{*}(H)=G$ for a given graph $G$.
\end{abstract}

Keywords: radius, diameter, super-radial graph.

2010 Mathematics Subject Classification: 05C12. 


\section{REFERENCES}

[1] J. Akiyama, K. Ando and D. Avis, Eccentric graphs, Discrete Math. 56 (1985) 1-6. doi:10.1016/0012-365X(85)90188-8

[2] R. Aravamuthan and B. Rajendran, Graph equations involving antipodal graphs, presented at the Seminar on Combinatorics and Applications held at ISI, Calcutta during 14-17 December (1982), 40-43.

[3] R. Aravamuthan and B. Rajendran, On antipodal graphs, Discrete Math. 49 (1984) 193-195. doi:10.1016/0012-365X(84)90117-1

[4] R. Aravamuthan and B. Rajendran, A note on antipodal graphs, Discrete Math. 58 (1986) 303-305. doi:10.1016/0012-365X(86)90148-2

[5] F. Buckley and F. Harary, Distance in Graphs (Addition-Wesley, Reading, 1990).

[6] F. Buckley, The eccentric digraphs of a graph, Congr. Numer. 149 (2001) 65-76.

[7] E. Prisner, Graph Dynamics (Longman, London, 1995).

[8] G. Johns and K. Sleno, Antipodal graphs and digraphs, Internat. J. Math. Soc. 16 (1993) 579-586. doi:10.1155/S0161171293000717

[9] G. Johns, A simple proof of the characterization of antipodal graphs, Discrete Math. 128 (1994) 399-400. doi:10.1016/0012-365X(94)90131-7

[10] Iqbalunnisa, T.N. Janakiraman and N. Srinivasan, On antipodal eccentric and supereccentric graph of a graph, J. Ramanujan Math. Soc. 4(2) (1989) 145-161.

[11] J. Boland, F. Buckley and M. Miller, Eccentric digraphs, Discrete Math. 286 (2004) 25-29. doi:10.1016/j.disc.2003.11.041

[12] J. Gimbert, M. Miller, F. Ruskey and J. Ryan, Iterations of eccentric digraphs, Bull. Inst. Combin. Appl. 45 (2005) 41-50.

[13] J. Gimbert, N. Lopez, M. Miller and J. Ryan, Characterization of eccentric digraphs, Discrete Math. 306 (2006) 210-219. doi:10.1016/j.disc.2005.11.015

[14] KM. Kathiresan and G. Marimuthu, A study on radial graphs, Ars Combin. 96 (2010) 353-360.

[15] KM. Kathiresan and G. Marimuthu, Further results on radial graphs, Discuss. Math. Graph Theory 30 (2010) 75-83. doi:10.7151/dmgt.1477

[16] KM. Kathiresan, G. Marimuthu and S. Arockiaraj, Dynamics of radial graphs, Bull. Inst. Combin. Appl. 57 (2009) 21-28. 
[17] KM. Kathiresan and R. Sumathi, Radial digraphs, Kragujevac J. Math. 34 (2010) 161-170.

[18] KM. Kathiresan, S. Arockiaraj and C. Parameswaran, Characterization of supereccentric graphs, submitted.

[19] M.I. Huilgol, S.A.S. Ulla and A.R. Sunilchandra, On eccentric digraphs of graphs, Appl. Math. 2 (2011) 705-710. doi:10.4236/am.2011.26093

[20] N. López, A generalization of digraph operators related to distance properties in digraphs, Bulletin of the ICA 60 (2010) 49-61.

[21] R.R. Singleton, There is no irregular Moore graph, Amer. Math. Monthly 75 (1968) 42-43. doi: $10.2307 / 2315106$

[22] D.B. West, Introduction to Graph Theory (Prentice-Hall of India, New Delhi, 2003).

[23] X. An and B. Wu, The Wiener index of the $k^{\text {th }}$ power of a graph, Appl. Math. Lett. 21 (2008) 436-440. doi:10.1016/j.aml.2007.03.025

Received 25 June 2013 Revised 28 October 2013 Accepted 4 December 2013 